\title{
Novel satellite positioning system using Acousto-Optic Deflector
}

Mitsuru Musha

Kei Akami

Aru Suemasa

Institute for Laser Science Univ. of Electro-communications

ICSO2018, 12 ${ }^{\text {th }}$ Oct. 2018 @ Crete, Greece 


\title{
Outline
}

Gravitational wave

Space gravitational wave detector DECIGO/B-DECIGO

Satellite positioning system

\author{
Basic principle \\ Experimental setup \\ Resolution and performance
}

Conclusions 


\section{- Gravitational Wave Detection}

Gravitational Wave (GW)

Motion of Mass

inspiral, merger of $\mathrm{BHB}$ explosion of super nova predicted in 'General Theory of Relativity'

Ripples in gravitational field $\cap 0 \ominus \theta \theta$

Propagate as waves

First direct detection of GW by adv.LIGO
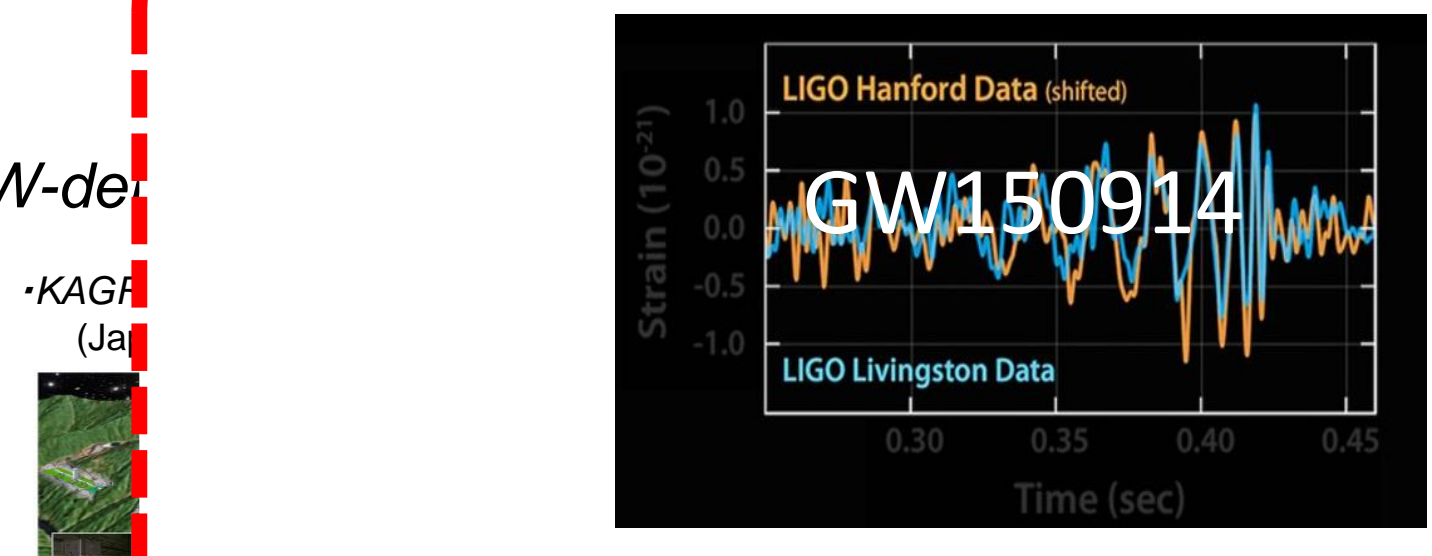

GW-de

$\cdot K A G A$

- GEC

(Ger

Verification of GW

Birth of GW astronomy
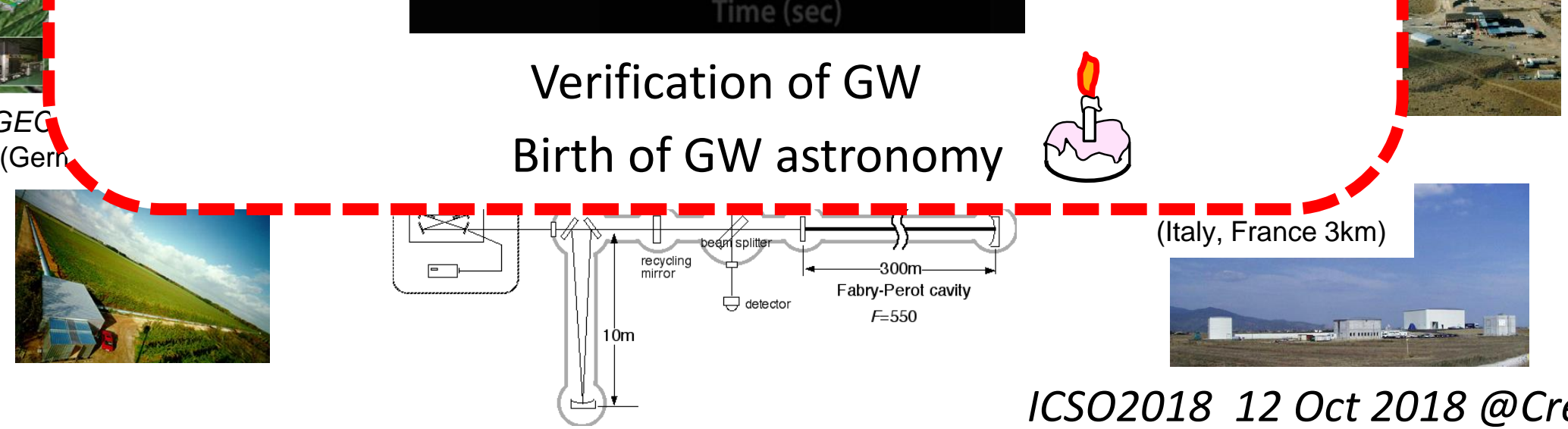
Arm length

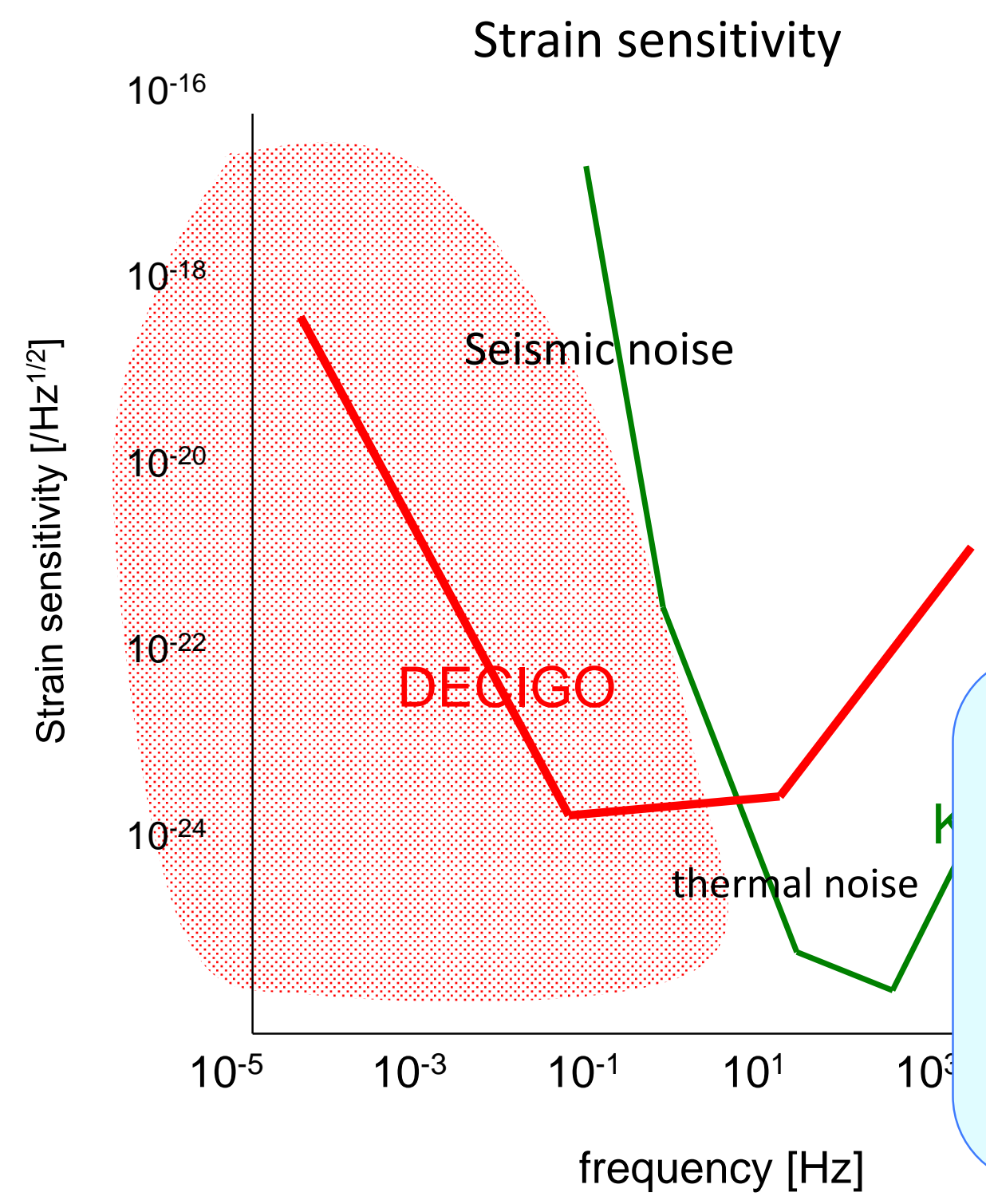

\section{Strain sensitivity}

Lower frequency range (attractive GW sources)

Seismic noise free longer Arm length

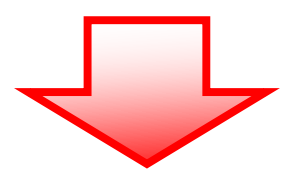

GW detector in space

\section{Space GW Detector}
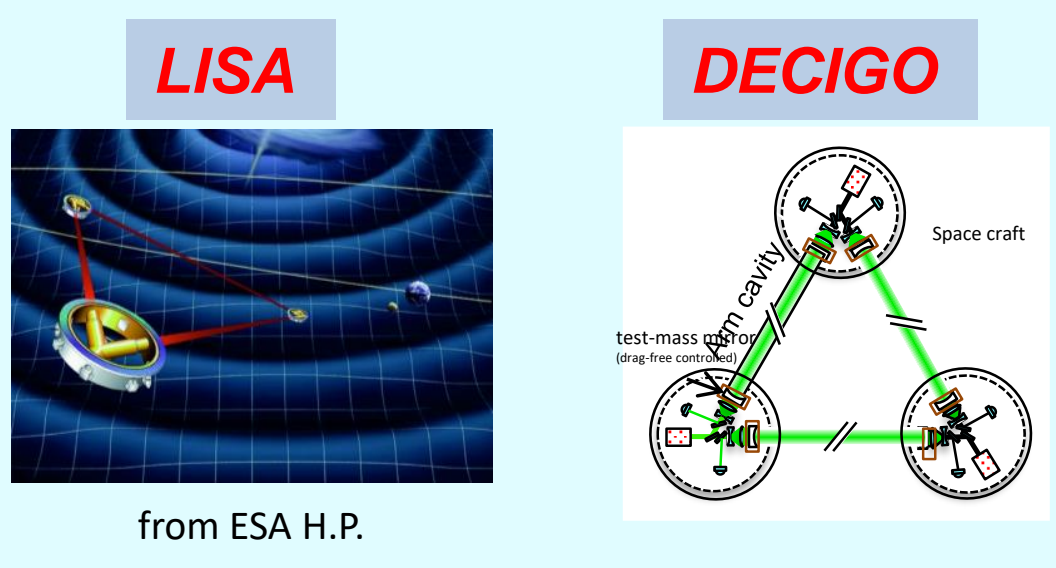


\section{DECIGO}

DECIGO (DECi-hertz Interferometer Gravitational-wave Observatory)

Differential Fabry-Perot interferometer

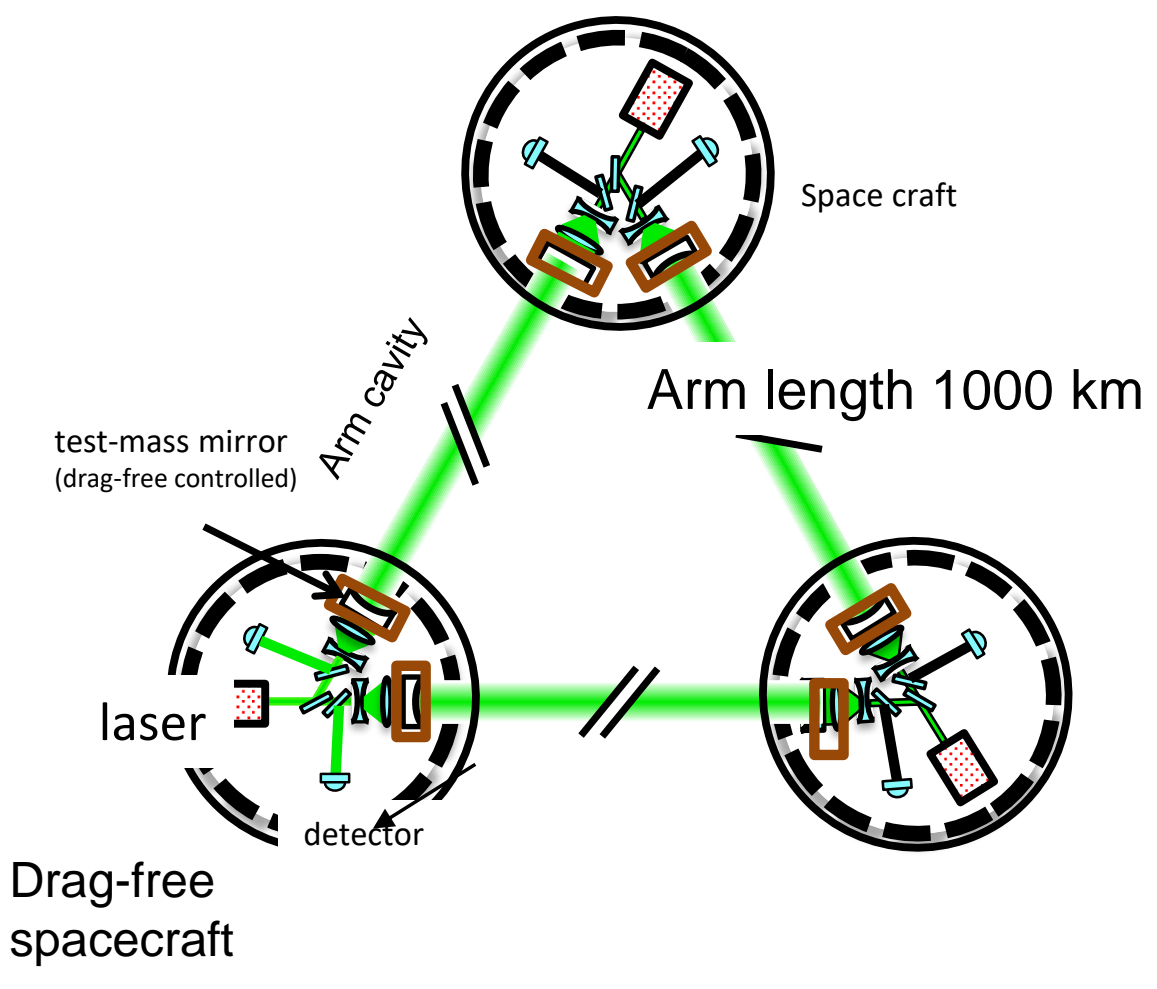

launched: mid 2030s
- $3 \mathrm{~S} / \mathrm{C}$ formation flight

- 3 Fabry-Perot interferometer

$$
\begin{aligned}
& L=1000 \mathrm{~km} \\
& F=10
\end{aligned}
$$

- mirrors $w=100 \mathrm{~kg}$.

$$
\Phi=1 \mathrm{~m}
$$

- Drag-free control

- laser power 10W@0.5 $\mu \mathrm{m}$

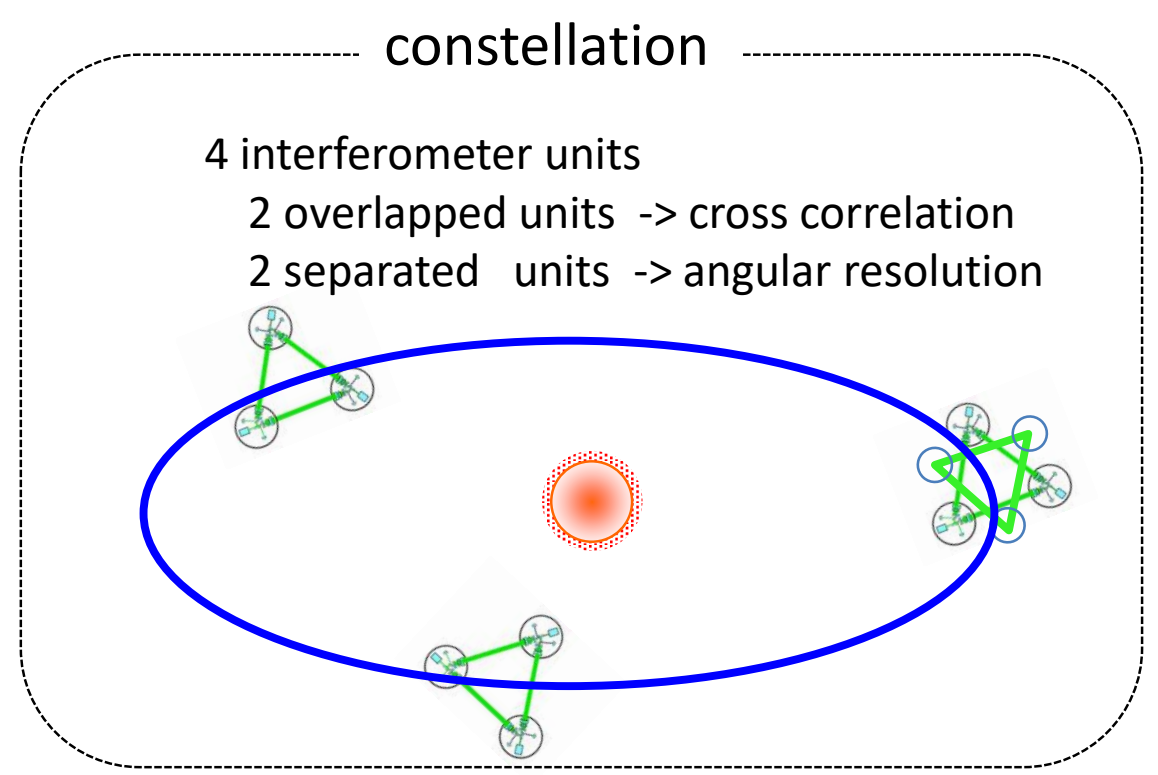




\section{- Key technologies for DECIGO}

Highly-stable high power light source

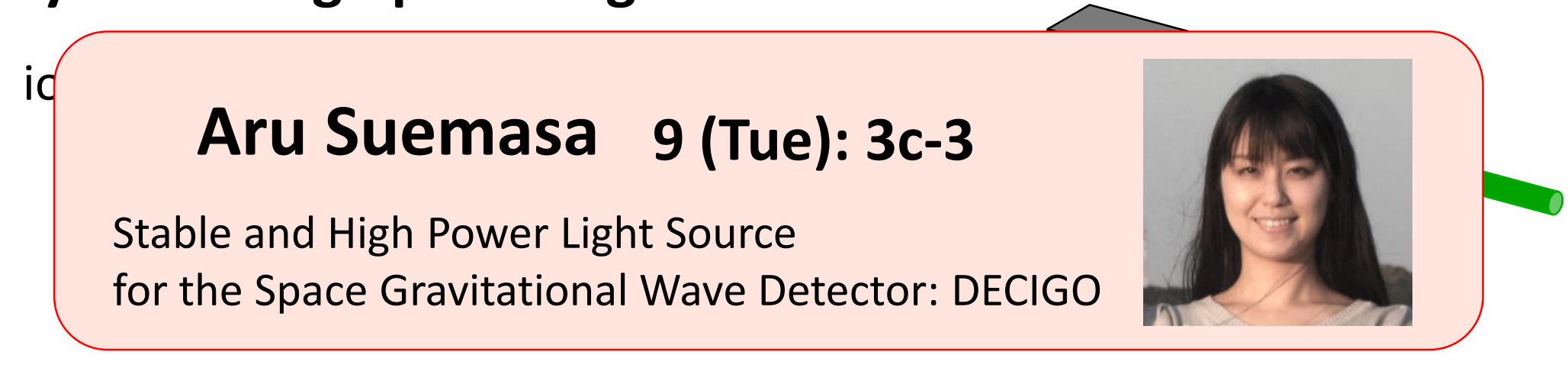

Precision satellite positioning system

Initial alignment of $3 \mathrm{~S} / \mathrm{C}$

maintaining of the formation flight

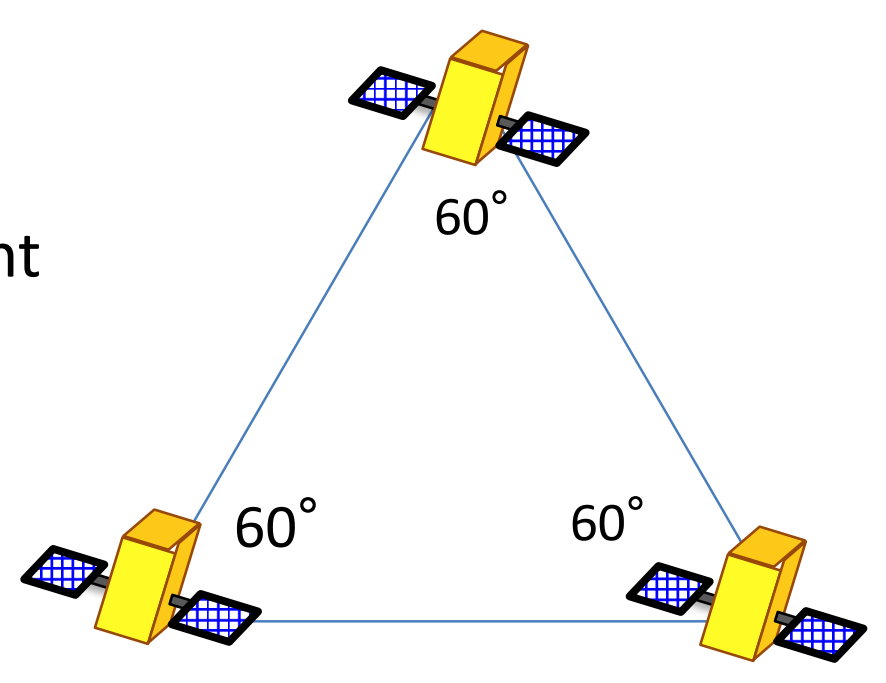

$\delta L / L \sim 0.5 \%$ 


\section{DECIGO}

\section{Satellite tracking}

$\Delta$

initial alignment keeping triangle shape

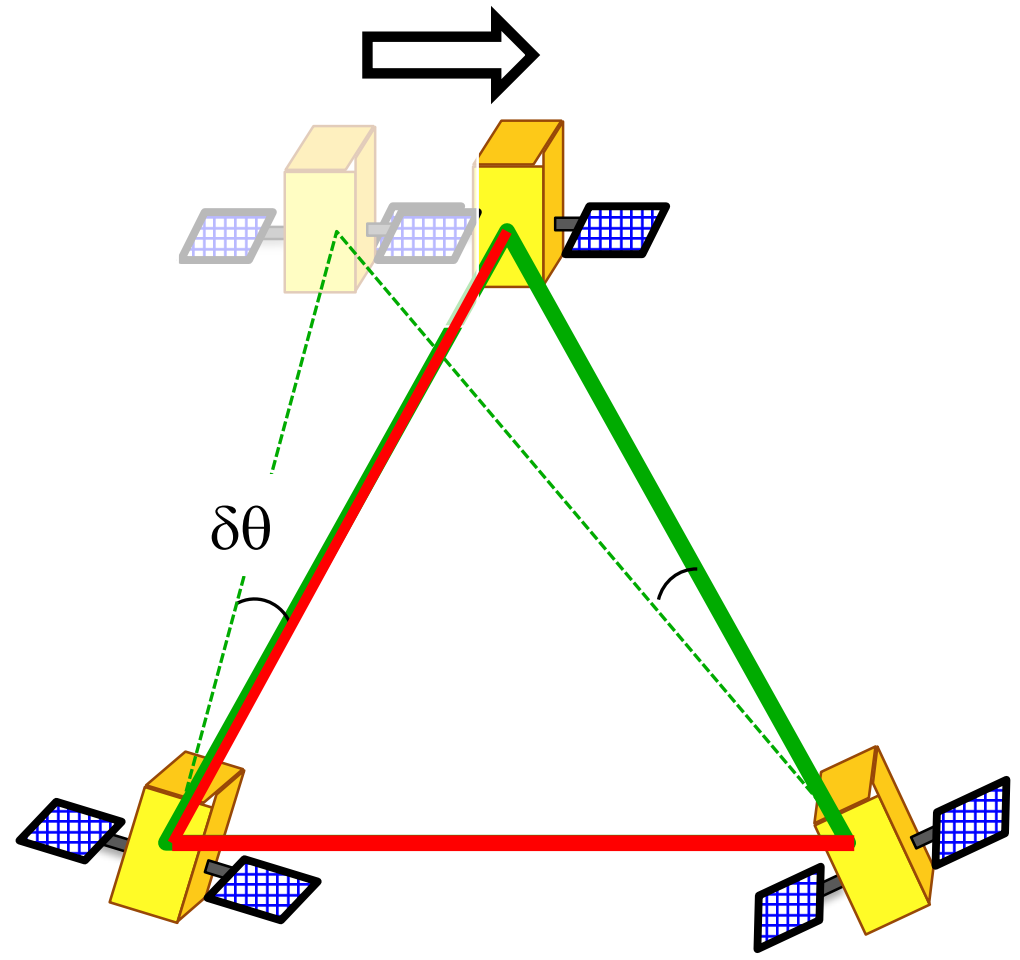

Let them know the angle error

Inter-satellite optical communication

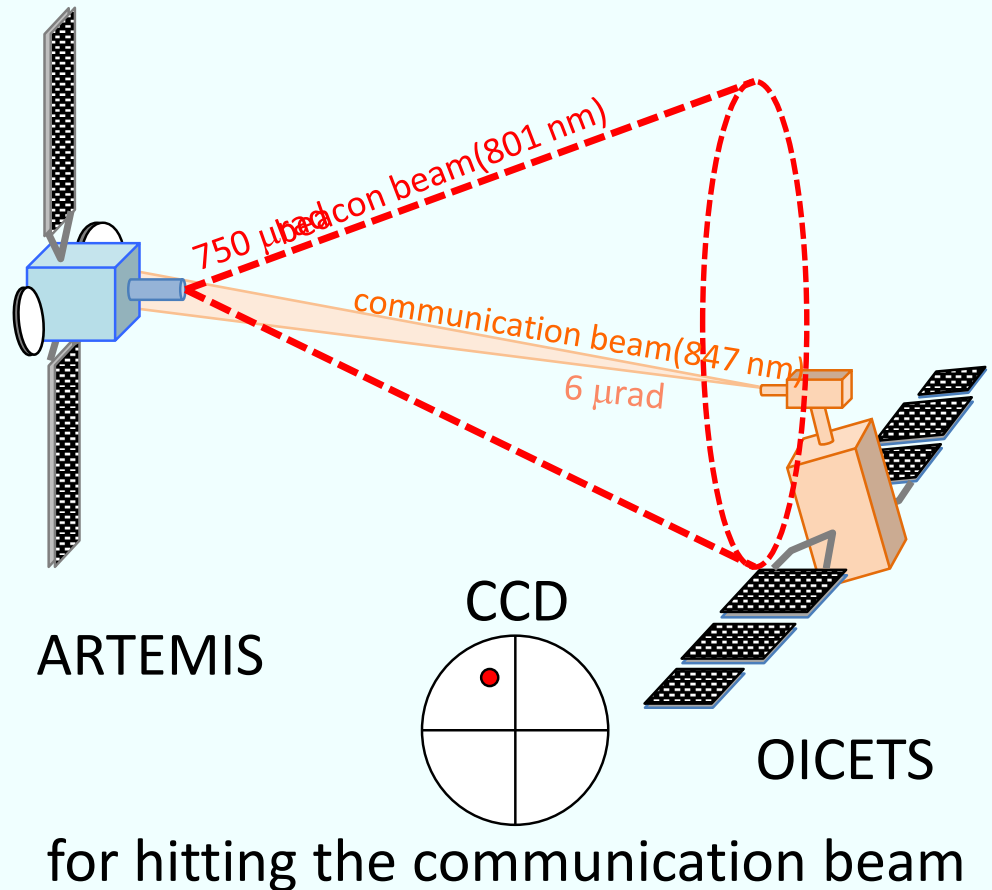

for hitting the communication beam 


\section{Acousto-Optic Deflector : AOD}

Diffraction angle is proportional to $f_{m}$

$1^{\text {st }}$ diffraction beam is frequency-shifted by $f_{m}$

$$
=\frac{f_{m}}{V_{a}} \quad \begin{aligned}
& \lambda: \text { wavelength } \\
& \mathrm{v}_{\mathrm{a}}: \text { Acoustic velocity }
\end{aligned}
$$

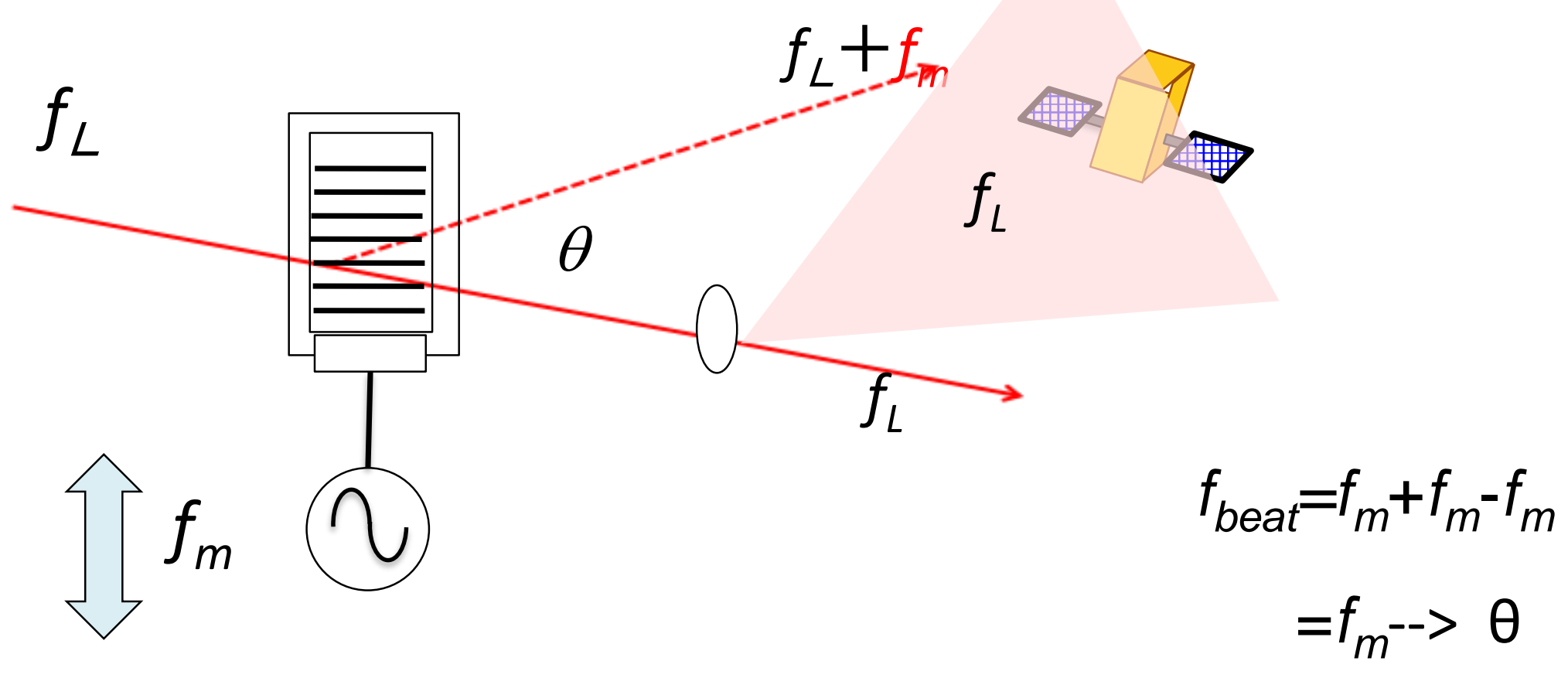




\section{Experimental setup}

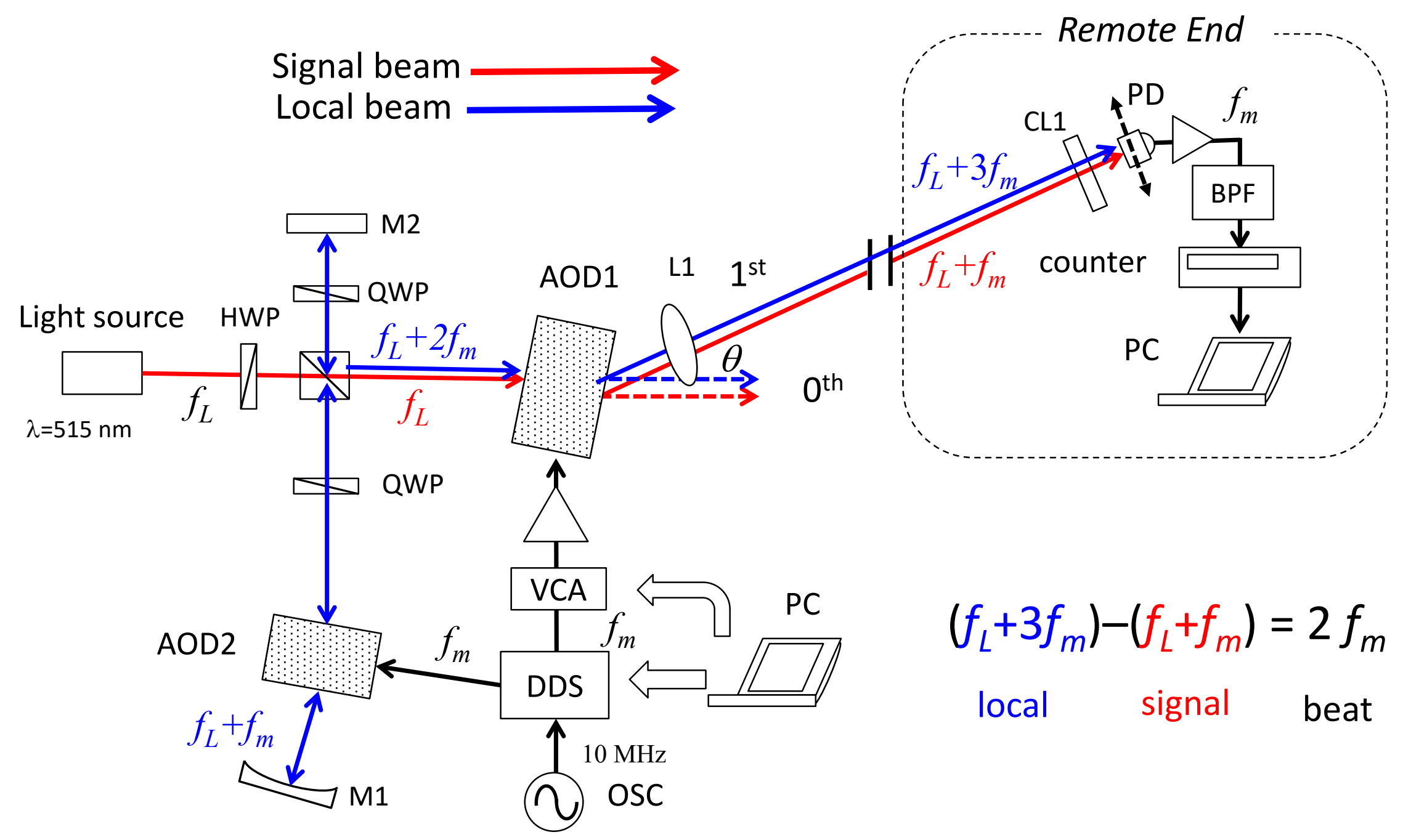


Advantages

High spatial resolution from freq. counting

No mechanical movements

Compactness

Free from frequency fluctuations of the light source

feasibility

Spatial resolution

Required detected power

Scanning speed

Scanning range 


\section{Optical spatial resolution}

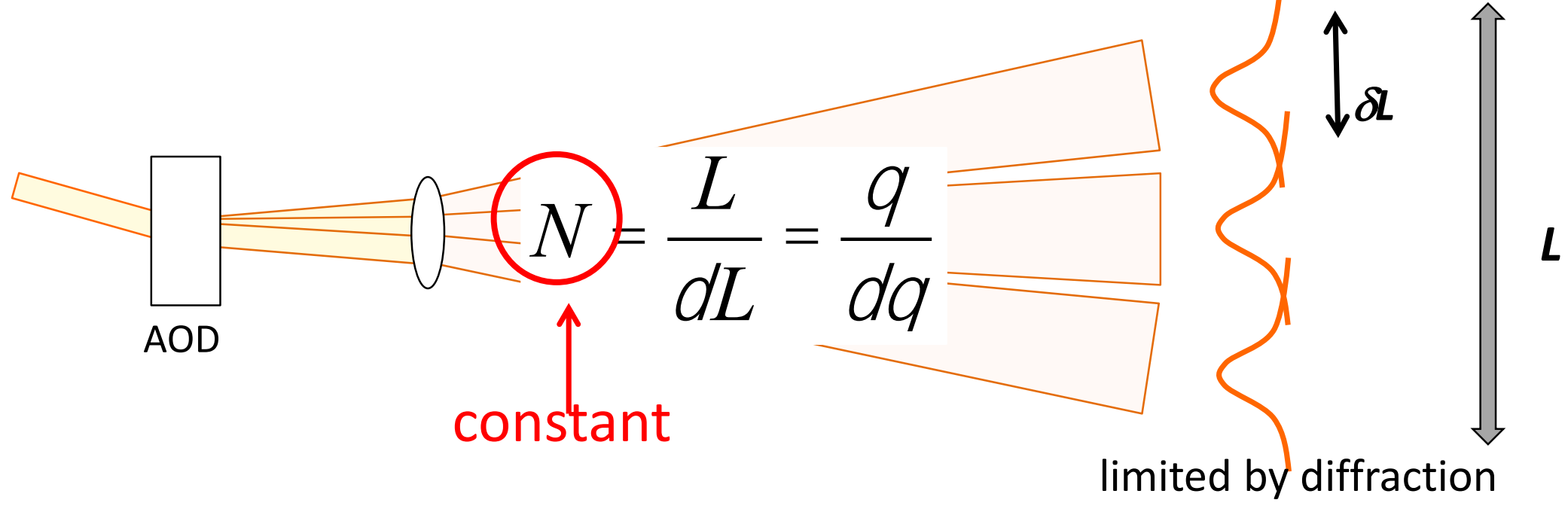

\section{Frequency counts}
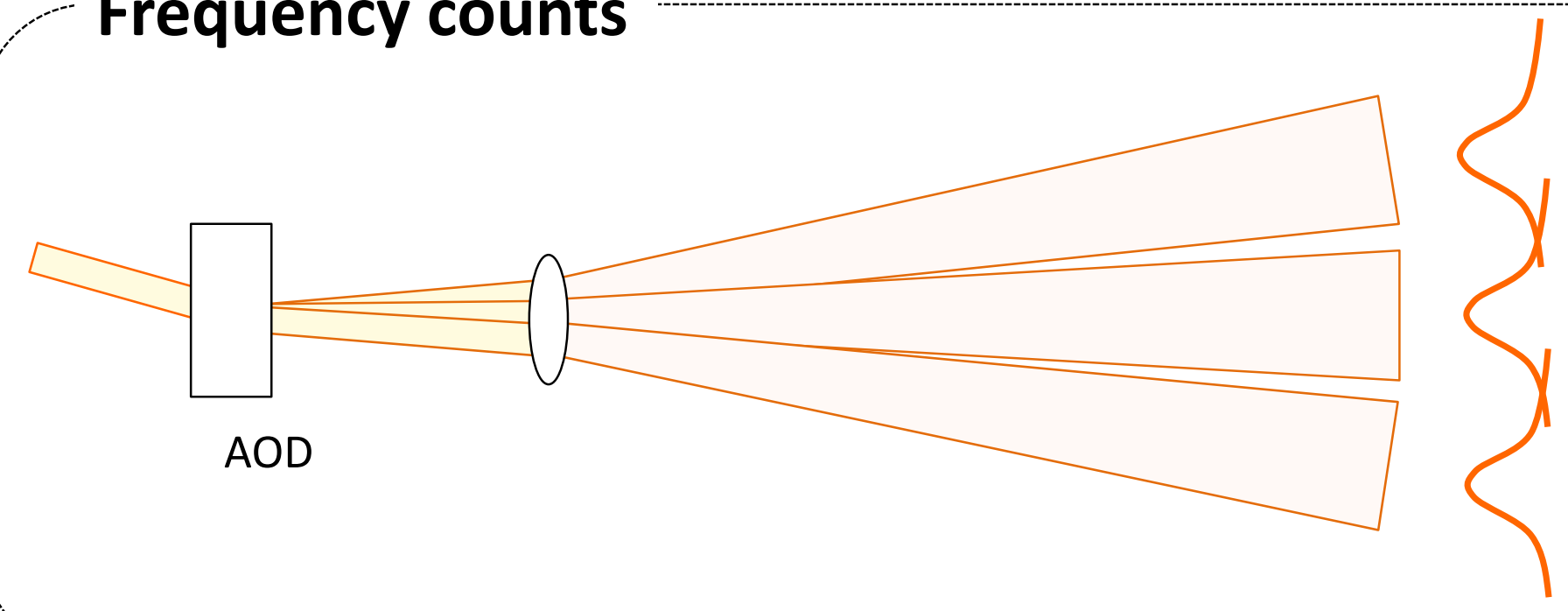

Aperture

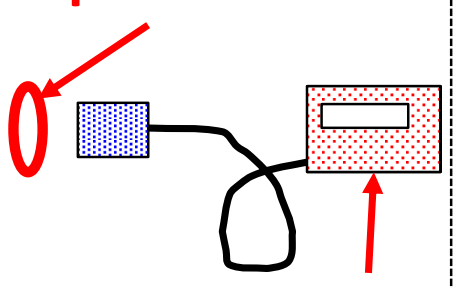

resolution 
beam diam. > aperture

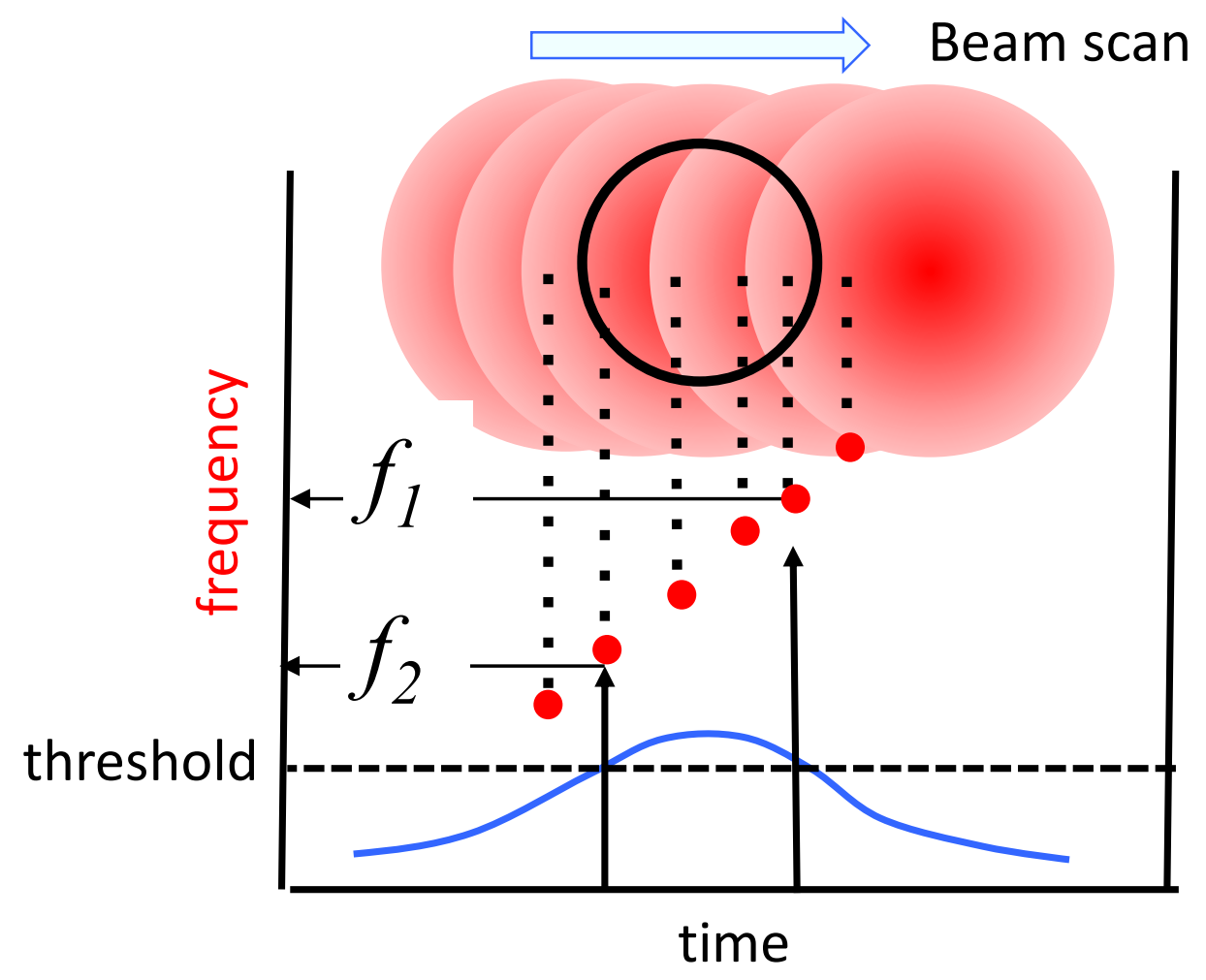

frequency at sweeping edge

(above certain threshold)

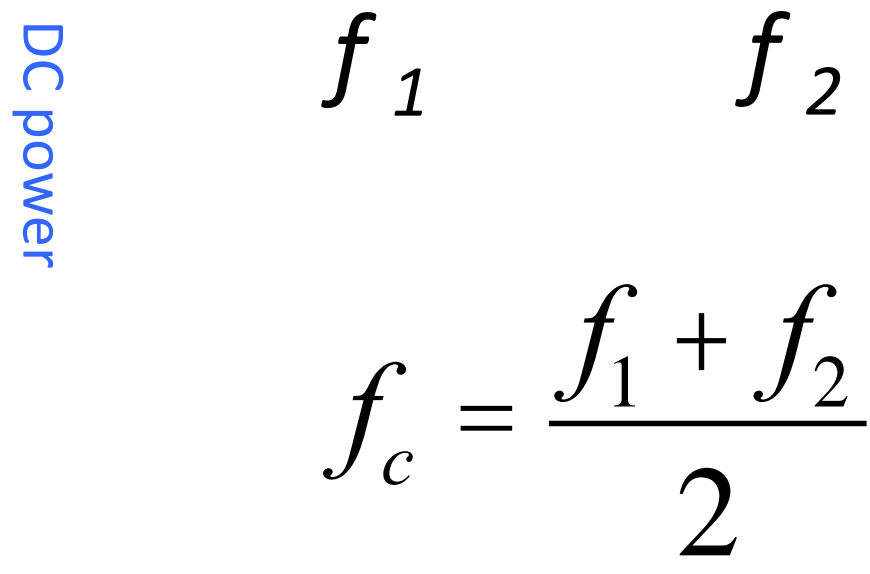

freq. at middle point 


\section{Spatial Resolution}

spatial resolution test

remote

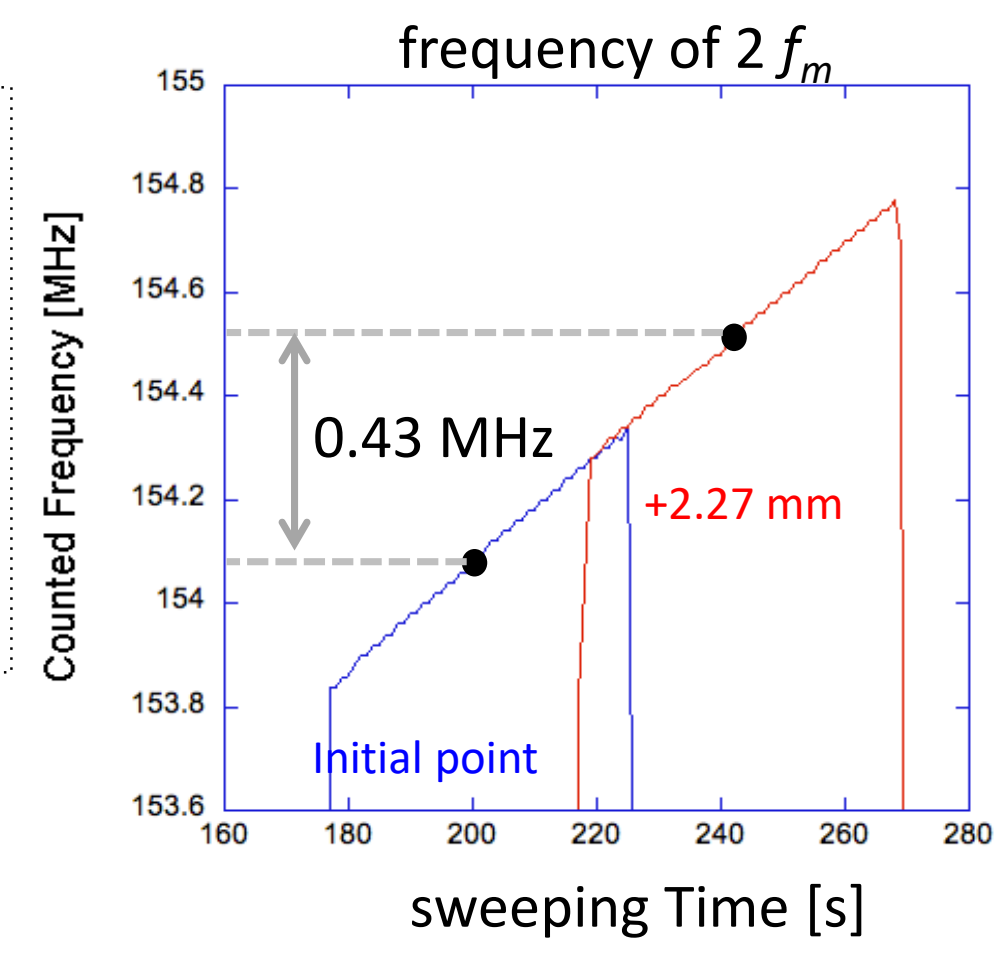

with power stabilization of diffracted light

$$
\begin{aligned}
\delta f= & 0.03 \mathrm{MHz} \\
N_{\text {exp }}= & 1666 \hookleftarrow 108\left(N_{\text {opt }}\right) \\
& \text { x16 improvement }
\end{aligned}
$$

\begin{tabular}{|l|c|c|}
\hline & set & meas. \\
\hline$\delta \theta[\mathrm{MHz}]$ & 0.46 & 0.43 \\
\hline
\end{tabular}

\begin{tabular}{|c|c|c|}
\hline & opt & freq. \\
\hline res: $\mathrm{N}$ & 108 & 1666 \\
\hline
\end{tabular}

x10 improvement 


\section{necessary conditions}

\section{- Detection power \\ - Scanning Speed}

\section{resolution $>1 \mathrm{~m} \quad: 10 \mathrm{kHz}$}

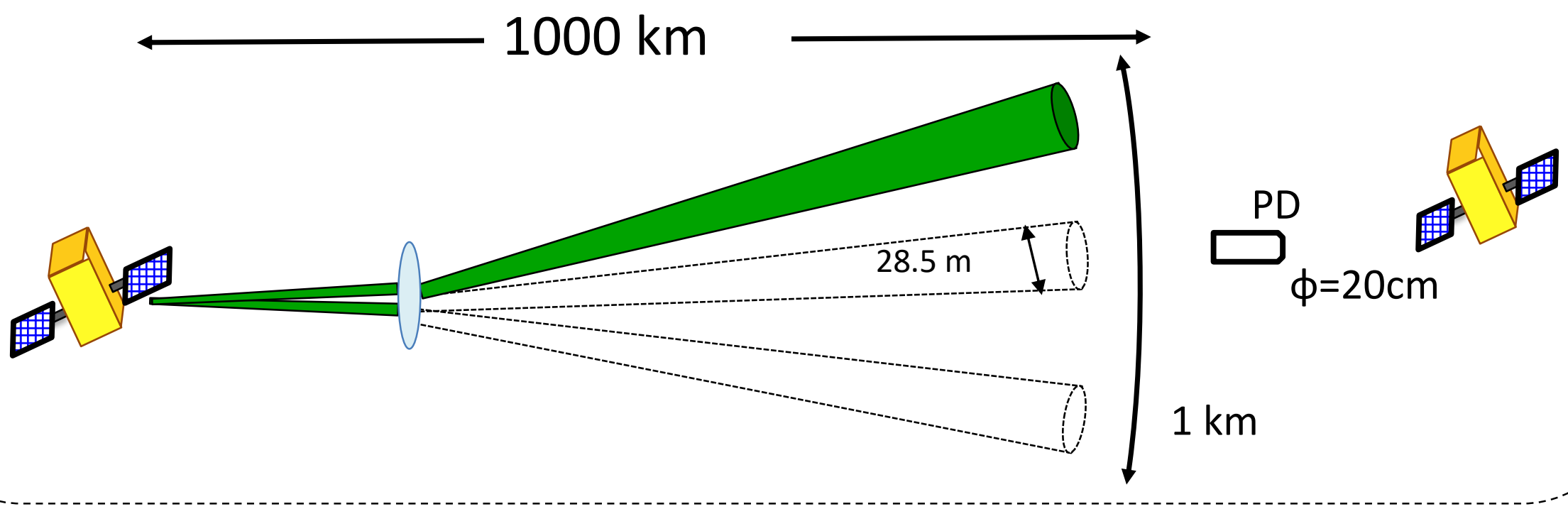




\section{Detection power}

- Detection power

-> SNR $->f$ resolution

$f_{\text {res }}$ vs $P_{\text {det }} \quad 10 \mathrm{kHz} \rightarrow 5 \mu \mathrm{W}$

$P_{\text {det }}$ vs $P_{\text {source }} \quad 5 \mu \mathrm{W} \rightarrow 98 \mathrm{~mW}$

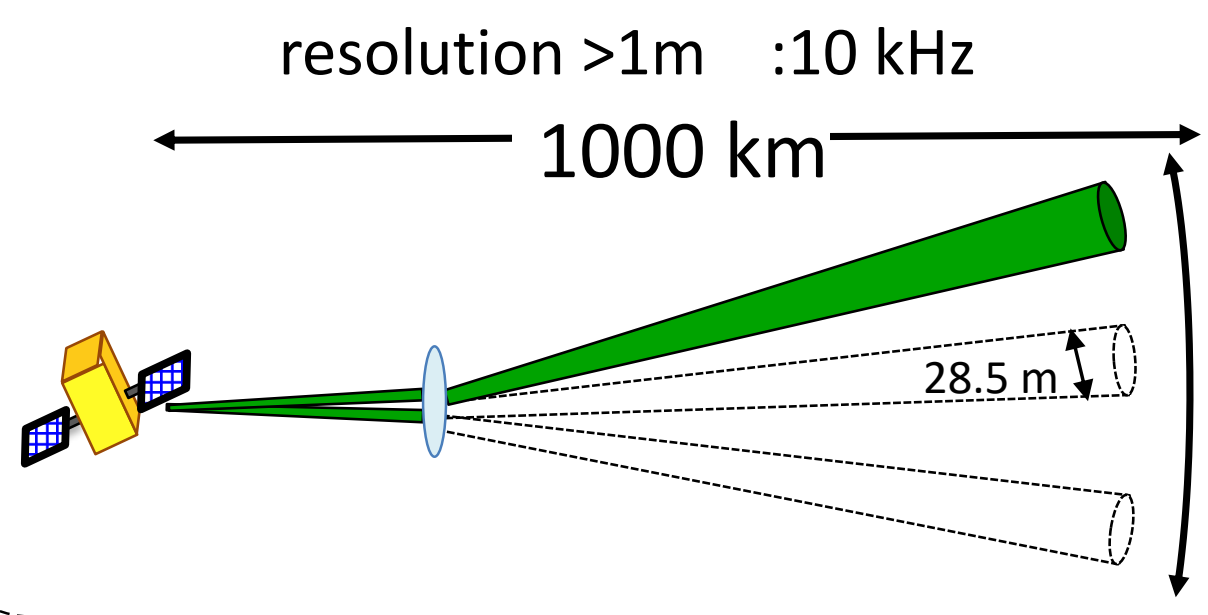

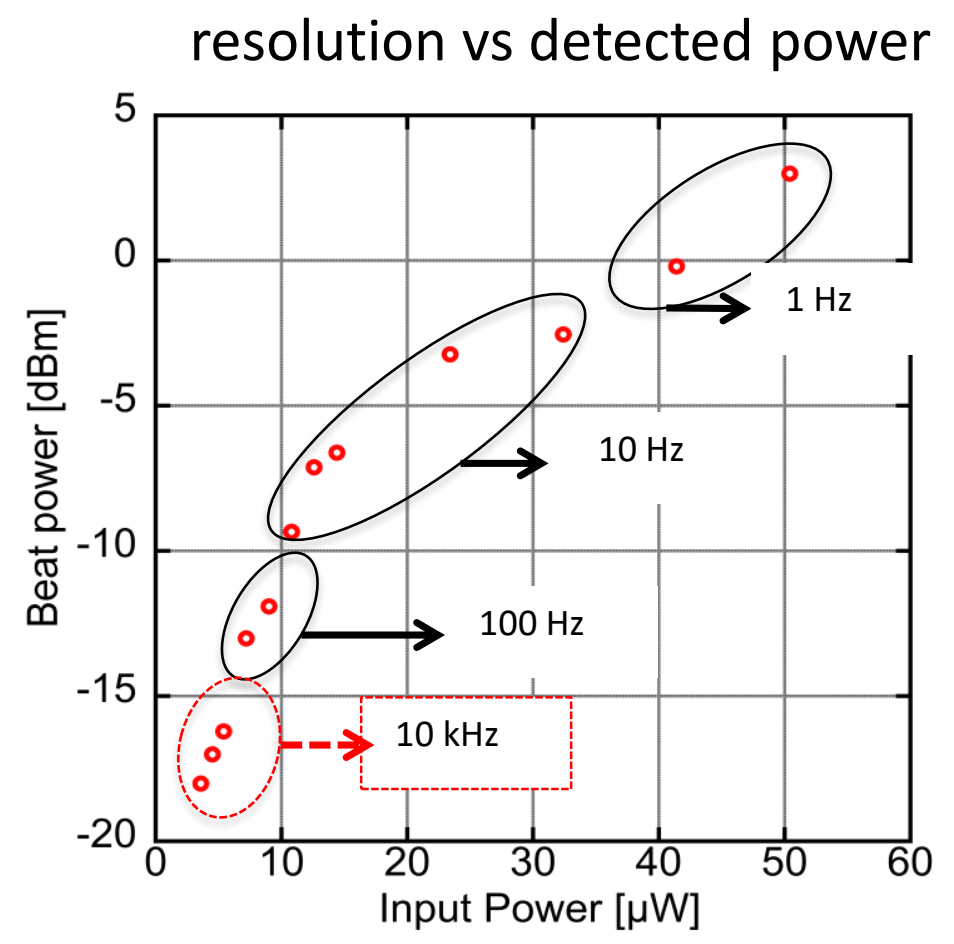

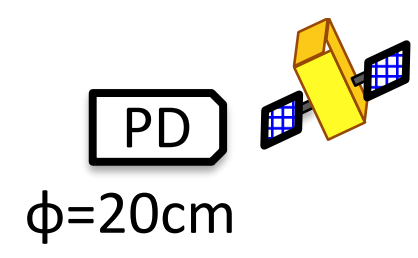

$1 \mathrm{~km}$ 


\section{Scanning speed}

- Scanning speed

$->$ detection time

gate time $\tau=1 \mathrm{~ms}(100 \mathrm{~Hz})$

detected time $<40 \mathrm{~ms}$

sweeping time $->3 \mathrm{~s}$

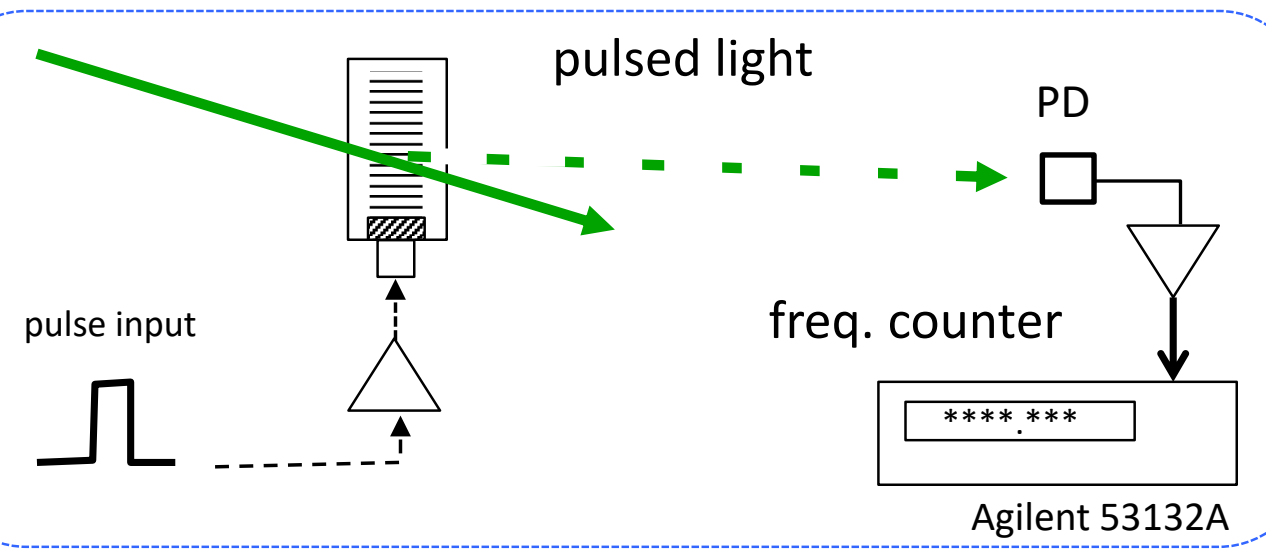

optimizing counting condition

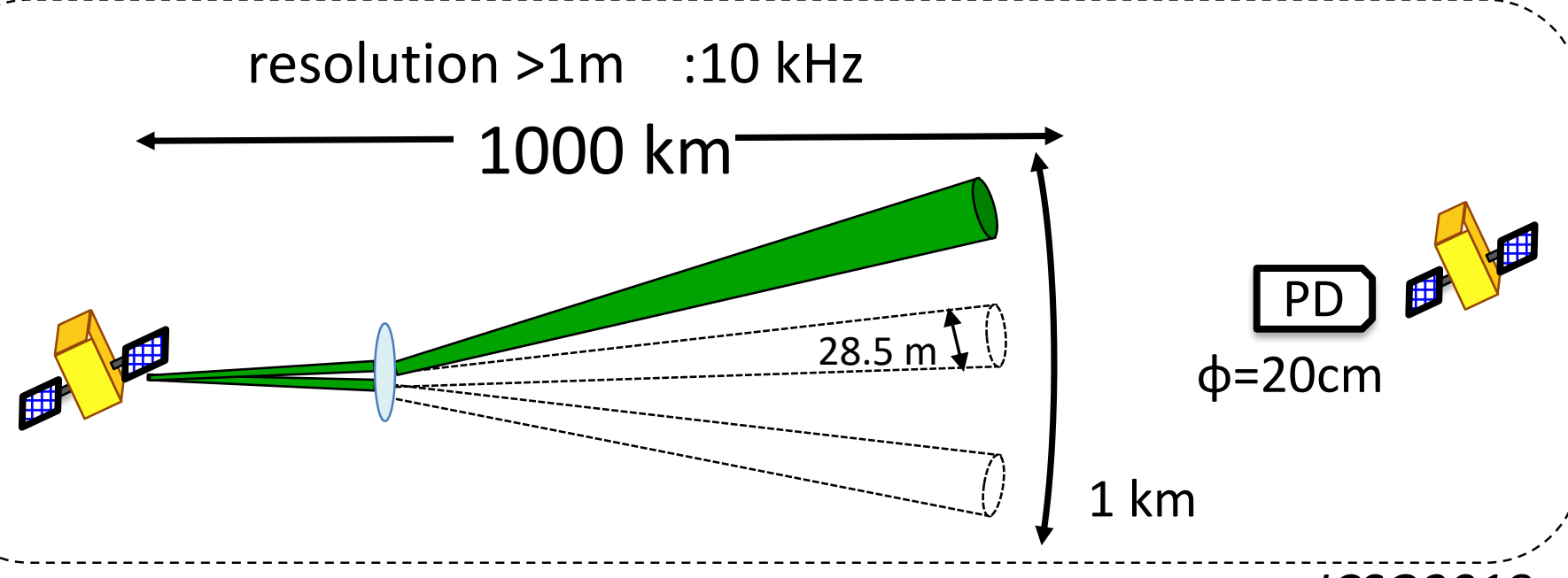


Novel satellite positioning system based on AOD

higher angle resolution $\quad \times 16$

fast scanning

required power $100 \mathrm{~mW}$

sweeping time $3 \mathrm{~s}$

\section{future prospects}

*further precision feasibility test

long-distance experiment

fast scanning

2-D scanning

1 AOD system

Course-fine scanning 
This research is supported by

the Japan Aerospace Exploration Agency (JAXA)

the Japan Society for the Promotion of Science (JSPS)

Grand-in-Aid for Scientific Research

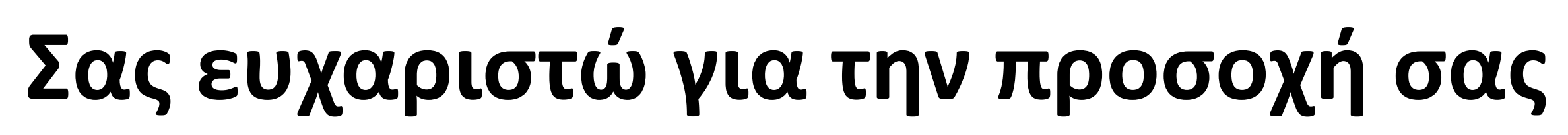




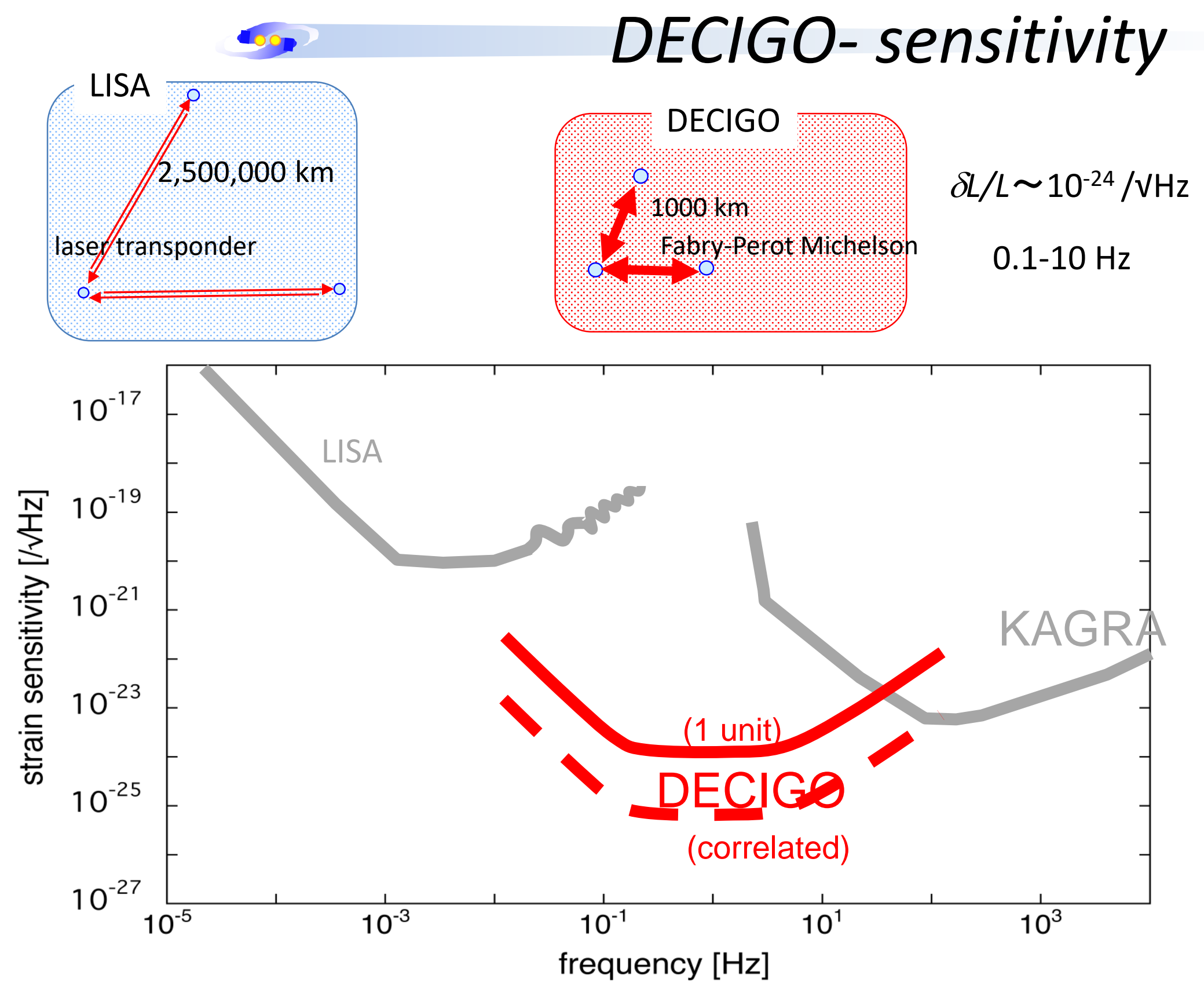

ICSO201812 Oct $2018 @$ Crete 


\section{DECIGO-targets}

NSB-inspiral

O IMBHB-inspiral

Formation mechanism of SMBH

Galaxy formation

Cosmology (inflation, dark energy)

O Stochastic background from very early universe

Birth of Universe

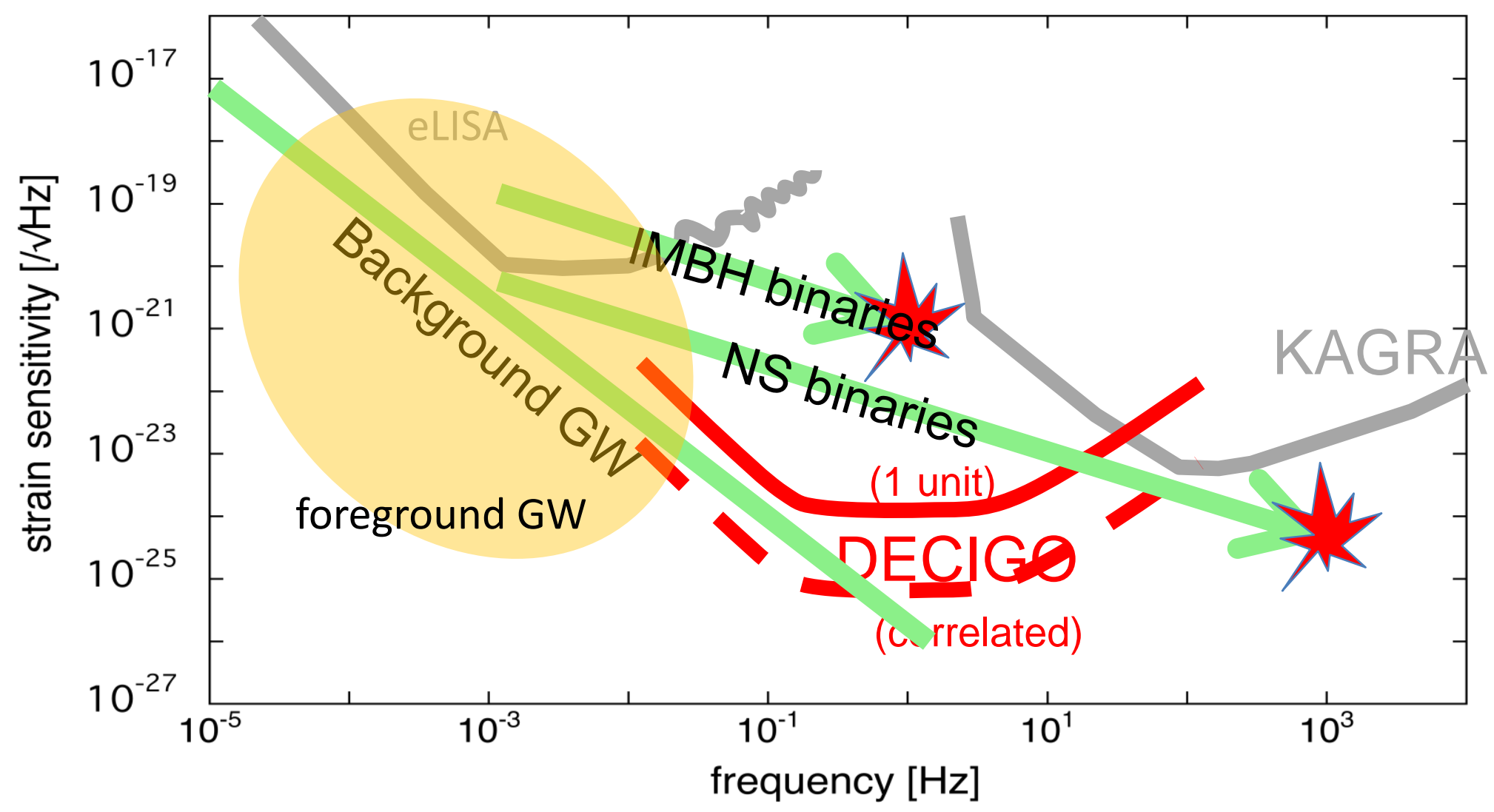

ICSO201812 Oct $2018 @$ Crete 